\title{
Exploring Mathematical Concepts in Literature
}

\author{
Christopher M. Kribs Zaleta \& Kim K. Ruebel
}

"We live in a mathematical world," begins the Principles and Standards for School Mathematics: An Overview

(National Council of Teachers of Mathematics 2000).

The document goes on to discuss how those lacking in math skills will be left out of important opportunities in life. In fact, they say, mathematical knowledge leads to the opening of doors, careers, and overall fulfillment in our personal lives. These statements are fascinating from another perspective- that of a reading teacher. Many a reading teacher's philosophy focuses on these same key arguments. We live in a world in which reading is essential. Literature opens doors-doors to our minds, worlds that may otherwise be inaccessible, job opportunities, and, of course, personal fulfillment. If "literature speaks to the heart of the child" (Spann, 1992) and mathematics speaks to the mind, then surely there are ways to use their intersections and children's interest in one to generate interest in the other by finding and exploring mathematical concepts as they are embedded in stories and other literature. According to Carol Hurst, "We're talking about blurring the edges of math and literature and letting children play with math concepts without destroying the work of literature that can lead to those concepts or extend them." Hurst's
Children's Literature Site is filled with ideas for linking literature with other subjects (www.carolhurst.com). Most of the information, reviews, and ideas are geared toward the elementary grades, with a few to the fourth through sixth grade. However, more than a few ideas and books for middle schoolers are described in this article.

Much has been written on the subject of linking math and literature. However, the focus is usually on the incorporation of reading into a math class-how a teacher might use picture books, problems, or stories, in general, to bring enthusiasm and engagement into the math classroom. We propose that teachers think about linking these two subjects in the opposite way, incorporating math into a literature class as a means to build comprehension and enthusiasm and as a way to discover mathematical concepts as they exist in literature. Literature can provide a way to make mathematics learning more personal. However, since children learn best when knowledge takes on an "immediate importance and purpose" (Beane, 1997, p. 2) and when learning has meaning and relevance, could we not argue the inverse as well that mathematics can provide a way to make literature much more personal? Using mathematics to explore new scales

This article reflects the following This We Believe characteristics: Students engaged in active learning - Curriculum that is relevant, challenging, integrative, and exploratory - Multiple learning and teaching approaches that respond to student diversity 
and perspectives has the potential to improve reading comprehension. The literature connection provides a context for using mathematics to solve problems (Jacobs \& Rak, 1997; Melser \& Leitze, 1999).

The instructional processes of inquiry and problembased learning are essential to the implementation of the ideas presented here. Key to this idea of math inside the literature classroom is an awareness and understanding of what math concepts children at each grade level need to be developing. So, before jumping into the study of literature rich with mathematical concepts, let us define inquiry and problem-based learning to build a link between math and literature.

\section{Inquiry and problem-based learning}

Inquiry learning, requiring active involvement during the learning process, leads to understanding. Learners must seek answers to key questions, resolve issues, and solve problems while constructing new knowledge. Effective inquiry, however, is more than just asking questions. Students must find ways to turn data and information into useful knowledge. According to Bransford, Brown, and Cocking (1999), useful application of inquiry learning involves several factors: a context for questions, a framework for questions, a focus for questions, and different levels of questions. Welldesigned inquiry learning produces knowledge that can be widely applied in other subjects and in real life.

In the spirit of promoting quality thinking using the inquiry method, problem-based learning has become a popular teaching approach. Problembased learning (PBL), at its most fundamental level, is an instructional method characterized by the use of "real-world" problems as a context for students to learn critical thinking and problem-solving skills and acquire knowledge of the essential concepts of a course (Albanese \& Mitchell, 1993; Engel, 1991). Using PBL, students acquire lifelong learning skills, which include the ability to find and use appropriate learning resources. Teachers guide, probe, and support students' initiatives but do not lecture, direct, or provide easy solutions. The NCTM standards state (NCTM, 2000, p. 11):

Systematic reasoning is a defining feature of mathematics. Exploring, justifying, and using mathematical conjectures are common to all content areas and, with different levels of rigor, all grade levels. Through the use of reasoning, students learn that mathematics makes sense. Reasoning and proof must be a consistent part of student's mathematical experiences in pre-kindergarten through grade 12 .

Take out the references to math as a subject, and this could be part of a justification for the International Reading Association or the National Council for Teachers of English standards. Much of the reader response movement, for instance, has included a push for exploring literature to make inferences and draw conclusions, all based on evidence and justification.

Reasoning and problem solving are an integral part of all mathematics and language arts learning. In everyday life and in the workplace, being able to think critically to solve problems is a necessary skill. NCTM goes further by saying that solving problems is not only a goal of learning mathematics but also a major means of doing so. Problem solving should not be an isolated part of the curriculum but should involve all content areas. We believe this to be a prime argument for incorporating mathematical concepts into a language arts class, which can be a motivator for kids who are good at math but lack reading motivation. But more than just for motivation, relevant problems, patterns, and other phenomena can be investigated as they occur in literature, without being forced. Melser and Leitze (1999) wrote, "By integrating language arts and mathematical problem solving, we believe we can successfully harness a teacher's enthusiasm for and creativity in the language arts, then use that enthusiasm and creativity to further students' mathematical problem-solving skills.” Mathematical problem-solving activities "build upon and reinforce comprehension in language arts instruction ... particularly when problemsolving activities require the student to refer to the story for additional information that is necessary to solve the problem" (Melser \& Leitze, 1999). Even further, connecting language arts and mathematics "helps to open students' eyes to the work of mathematical thinking that

Figure 1 Children's books cited

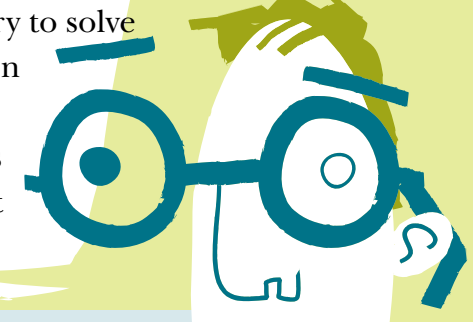

Adams, R. (1972). Watership down. New York: Macmillan.

Babbitt, N. (1975). Tuck everlasting. New York: Farrar, Straus \& Giroux.

Juster, N. (1964). The phantom tollbooth. New York: Random House.

Sachar, L. (1998). Holes. New York: Farrar, Straus \& Giroux 
surrounds everyday storybook characters, thereby making mathematics seem more relevant to their lives" (p. 48).

Pasko (2004) found, in her action research with young adolescents, that "having students make connections in learning not only helps with curricular and time demands, it allows learners to make meaning of material and enables them to retain information and/ or concepts learned." In her research, Pasko investigated the number, types, and accuracy of mathematical connections her students made in literature. For three weeks Pasko allocated the independent reading time in literature class to students' trying to find math concepts in their books. Students documented their findings on sticky notes and in a journal as they read. Pasko found that her students were, after some initial practice, able to identify such connections fairly accurately, but left open the question of whether students would do so without prompting. Jenner (2002) suggested that they do. Pasko found further support for making connections between these subjects in the work of Kliman (1993) and Hellwig, Jacobs, and Monroe (2000).

Bintz and Moore (2002) argued for organizing texts about related mathematical ideas so that teachers can use the literature to make mathematics more engaging and relevant to their students, who come to mathematics classrooms with diverse backgrounds. We argue that mathematical ideas and concepts can be used to make literature more engaging and relevant to the students. In this article we use specific examples to illustrate some of the significant intersections of middle grades literature and mathematics.

\section{Middle level mathematical concepts}

Middle school is the setting for the beginning of an important transition into intellectual adulthood. In the middle grades, students are beginning to grapple with significant abstract issues and to judge themselves, their peers, their parents, teachers, and the world around them. This urge to be critical is accompanied by an increased ability to handle details, to consider the fairness or unfairness of a situation, or to determine the reasonableness of an idea. They have within their grasp the basic mathematical tools for coming to quantitative terms with information, and so the mathematical discussions that come out of the literature they read should channel these impulses and abilities into inspection of the quantitative aspects of situations and judgments as to whether or not the results are reasonable.

In other words, they are moving from a simple awareness of the existence of quantitative facets of a scenario to investigation of the details. A recent NCTE policy brief recommended interpreting texts "that offer multiple perspectives on life experiences" and developing "capacities for critical thinking in each discipline" as among strategies that foster adolescent literacy (NCTE, 2006). Estimates of scale and the study of other perspectives are prime examples of areas in which numeracy can inform literacy. What follows are some examples of situations in which a mathematical investigation of some aspect of a story can help bring meaning to the story for students by placing them within its context.

\section{Estimation and probability}

In Louis Sachar's (1998) novel Holes, a boy named Stanley finds himself sent to a prison-like camp for juvenile delinquents located on the shore of a huge dry lake bed in rural Texas, where rehabilitation consists of each youth digging a hole every day that is five feet in each dimension. Stanley eventually discovers the warden's ulterior motive is to unearth a chest of stolen treasure dumped in the lake 90 years earlier. Throughout the story, Stanley complains of a family curse that has ruined his luck, yet in the end, Stanley is the one to find the chest. One can make some simple calculations to estimate the likelihood that Stanley (or indeed anyone) would find the treasure, to understand the magnitude of the warden's plan, and to see just how much Stanley's luck has to do with his discovery.

For example, we might estimate the number of holes it would take to dig up the fictional Green Lake, or how long it would take the camp residents to do so. To do this we must compare the area of the lake bed to the area of one hole, and figure the rate at which holes are dug. The fictional Green Lake was once "the largest lake in Texas." The real Green Lake, one of the largest natural freshwater lakes in Texas, is not dry and covers about 10,000 acres or about 440 million square feet. If we suppose that each hole dug measures five feet by five feet, we have an area of 25 square feet per hole. Dividing this into the area of the real Green Lake yields an estimate of about 17.5 million holes in the lake bed (assuming no space between the holes). If, instead, we require, say, two feet of space in between adjacent holes, 
so that they do not cave in on each other, then each hole marks off about 50 square feet, leaving room for about 8.25 million holes in the lake bed. Taking even this smaller figure as a conservative estimate, dividing by 365 holes/year/camper shows that it would take about 23,000 man-years to excavate the lake. Camp Green Lake has five tents, with six boys in each tent. Assuming continuous full occupancy, it would then take the camp more than 750 years to complete the excavation.

Even further reductions, restricting the search, for example, to the part of the lake bed nearest the original shore, will reduce the time required to finish digging up the lake bed by less than one order of
"Where could you ever find a number so big?" scoffed the Humbug.

"In the same place they have the smallest number there is," he answered helpfully; "and you know what that is." $[\ldots]$

"One one-millionth?" asked Milo, trying to think of the smallest fraction possible.

"Almost," said the Mathemagician. "Now divide it in half. Now divide it in half again. [...] Now divide it in half again. Now divide-"

"Oh dear," shouted Milo, holding his hands to his ears, "doesn't that ever stop either?"

"How can it," said the Mathemagician, "when you can

\section{He meets a partial child who claims to be the incarnation of the .58 part of the 2.58 children in the average family.}

magnitude, and the probability of any one person unearthing the treasure in a year's worth of digging holes remains around $365 / 825,000$ or $0.04 \%$. So, despite the family curse Stanley cites as ruining his luck, he was extraordinarily lucky to find the treasure-or instead, as the author suggests via the confluence of fates of the families described in the book, it was destiny.

\section{Infinity}

As children begin to grasp abstraction, one of the natural questions that arises involves the concept of infinity. Many children will ask what the largest number is long before they reach this age, and the simple reply that there is no largest number is unsatisfying without a context to provide intuition about why this should be so. Likewise, when the notion does arise in a story, it is constructive to have a discussion about what infinity really means-it is not a number, but an idea.

One story that affords such a discussion is Norton Juster's (1964) The Phantom Tollbooth, which has much of the whimsy and allegory of Alice in Wonderland. In this book, a boy named Milo visits the cities of Dictionopolis, the city of words, and Digitopolis, city of numbers. In the latter city, he has a conversation with the ruler about the largest number possible, which also leads into the question of the smallest number, in a manner reminiscent of Xeno's paradox: always take half of whatever you have left until it's so small that if you started to say it right now you'd finish even before you began?"

Shortly thereafter, Milo attempts to climb an infinite staircase, not surprisingly without much success. This episode not only provides further opportunity to discuss the notion of infinity, but that of statistical averages, when he meets a partial child who claims to be the incarnation of the .58 part of the 2.58 children in the average family.

"It must be rather odd being only part of a person," Milo remarked.

"Not at all," said the child. "Every average family has 2.58 children, so I always have someone to play with. Besides, each family also has an average of 1.3 automobiles, and since I'm the only one who can drive three tenths of a car, I get to use it all the time."

"But averages aren't real," objected Milo; “they're just imaginary."

This encounter is actually a powerful illustration of a rather complicated fact. When one hears of a statistical average, it is often easy to envision (at least, in cases where the average is reported as a whole number) many instances of the average describing the actual quantity present. For example, if we hear that the average family has enough garage space for one car, we may imagine blocks of houses with one-car garages. In fact, one-car garages are relatively rare; instead, most garages attached to houses are two-car garages, but there are also many 
families whose homes have no garages, and consequently the average would fall between the two. Milo's encounter with the statistical child brings out the problematic side to this simplistic approach, and may be confusing to children who have not given much thought to the idea of averages. A class discussion using another example based on data more readily visible to the students may help to clarify the idea-for example, the average number of pockets per person in the classroom.

The concept of infinity can manifest itself in other ways as well-perhaps less abstract, if no less fanciful. In Natalie Babbitt's (1975) Tuck Everlasting, a family of pioneers stumbles upon a spring whose water (they discover later) makes one effectively immortal. The Tucks have stopped aging and have been left untouched by they become a year older. Underlying this notion is the same concept as that discussed with regard to The Phantom Tollbooth: namely, that there is no single largest number.

\section{Other perspectives}

Richard Adams's (1972) popular novel Watership Down tells of an exodus of a group of rabbits from a doomed warren to a new home, safe in the hills of the English countryside. Among the many themes developed in the book (including the fact that the exodus is occasioned in the first place by urban development elbowing out nature), there are at least two for which a discussion involving mathematics can help young readers better understand and identify with the rabbits in the story: the distance of the journey itself and the number system the rabbits use.

\section{Although the Tucks will continue to get older, they will never reach a point where they will be infinitely old.}

accidents or events that should have proven fatal. Here the idea of living forever arises; the Tucks in particular are not happy with it, and a discussion of the story should include some exploration of exactly why the Tucks are so unhappy.

One way to explore the idea is to chart the ages of Miles Tuck and his family at different times. Although the years are not stated explicitly, save for a single line in the epilogue, it is not difficult to calculate from the data given that the story takes place in 1880, that the Tucks discovered the spring in 1793, and that Miles Tuck was born in 1771, Jesse in 1776 . With these starting points, one might assume, for example, that in 1795 Miles married a woman born in 1774 (21 years old), and that they had their two children in 1797 and 1800. One could make a chart listing the years in increments of 10, starting with 1800, and in other columns list the ages of Miles, his wife and children, with an additional column for Miles's apparent age (frozen at 22). After making the chart, the class can discuss at what point it would be difficult to believe that Miles had attained the age he had, and at what point Miles looked younger than his children.

If the students have had other discussions related to the idea of what infinity and forever mean, it might be constructive to point out that, although the Tucks will continue to get older, they will never reach a point where they will be infinitely old. Whatever their ages, every year
Most versions of the book include a scale map of the actual locality in England, near the town of Newbury, in Berkshire and Hampshire Counties, where the story takes place. For more details on the geography, or for those who do not have a map in their books, there are numerous reference sites available on the Internet, including Chris Boyce's "The Real Watership Down Page" (http://www. mayfieldiow.freewire.co.uk/watershp/). One can print and enlarge a map so that students can work on it to trace the path of the rabbits and later take measuring tapes and measure the length of their several-miles journey. Finally, they can make an analogy between the rabbits' size or length of one stride and the students' size or stride to determine an equivalent distance if the students were to make a journey of equal difficulty. For instance, if students estimate the rabbits' journey to be about seven miles, the rabbits' stride to be about nine inches, and their own stride to be about three feet (or 36 inches), an equivalent journey for the students would be four times seven miles, or 28 miles. Find a local map and identify some well-known location that is 28 miles from the school; tell students to imagine walking that distance by themselves (or as a class). Keep in mind that the rabbits' journey took several days, so the students' imaginary journey can also be divided up. One might also point out that the students will have to find new and different sources of food from their usual ones along the way. 
A discussion like this helps students put themselves in the rabbits' position and imagine the uncertainties they faced.

Another important insight into the way the rabbits thought can be obtained by considering their number system, first explained in a footnote in the first chapter. Rabbits count to four, but have only one word, "hrair," to denote any quantity larger than four. This word actually appears in two important names in the story: Fiver, actually Hrairoo, and the legendary rabbit hero El-ahrairah, translated as the Prince with a Thousand Enemies. In both cases, "hrair," rather than words meaning specifically five or 1,000, is used. The English number words are used to give readers an impression of the context, but this limitation on counting can itself yield important insights about rabbit mentality. One important question to ask is why the rabbits have no words for specific numbers larger than four. Certainly, one would expect a student to see a large difference between five and 1,000, and there are some obvious disadvantages from a human perspective in not being able to distinguish them. One response to the question might be that the rabbits are not able to count any further than four. Since one can imagine infant humans not yet able to count this high, this possibility is a reasonable one. However, another part of the answer is the rabbits' need for more number words: one can argue that their lives are such that they do not need them.

In fact, this kind of limited number system does exist in the world, and is or has been used in numerous aboriginal cultures of the Americas, Africa, and Australia. Burton (1997) wrote in The History of

\section{Mathematics: An Introduction:}

Anthropologists tell us that there has hardly been a culture, however primitive, that has not had some awareness of number, though it might have been as rudimentary as the distinction between one and two. Certain Australian aboriginal tribes, for instance, counted only to two, with any number larger than two called simply much or many. South American Indians along the tributaries of the Amazon were equally destitute of number words. Although they ventured further than the aborigines in being able to count to six, they had no independent number names for groups of three, four, five, or six. In their counting vocabulary, three was called two-one, four was two-two, and so on. A similar system has been reported for the Bushmen of South Africa, who counted to ten $(10=2+2+2+2+2)$ with just two words; beyond ten, the descriptive phrases became too long. It is notable that such tribal groups would not willingly trade, say, two cows for four pigs, yet had no hesitation in exchanging one cow for two pigs and a second cow for another two pigs. (pp. 1-2)

One might assert here, too, that such words will arise as are genuinely needed within the culture in question, and that, strange as it may sound, the rabbits had similar needs for being able to express quantity as these aboriginal cultures had. In any case, a consideration of the motivation behind the rabbits' number system in Watership Down can help illustrate for students the simplicity of this dimension of the rabbits' culture.

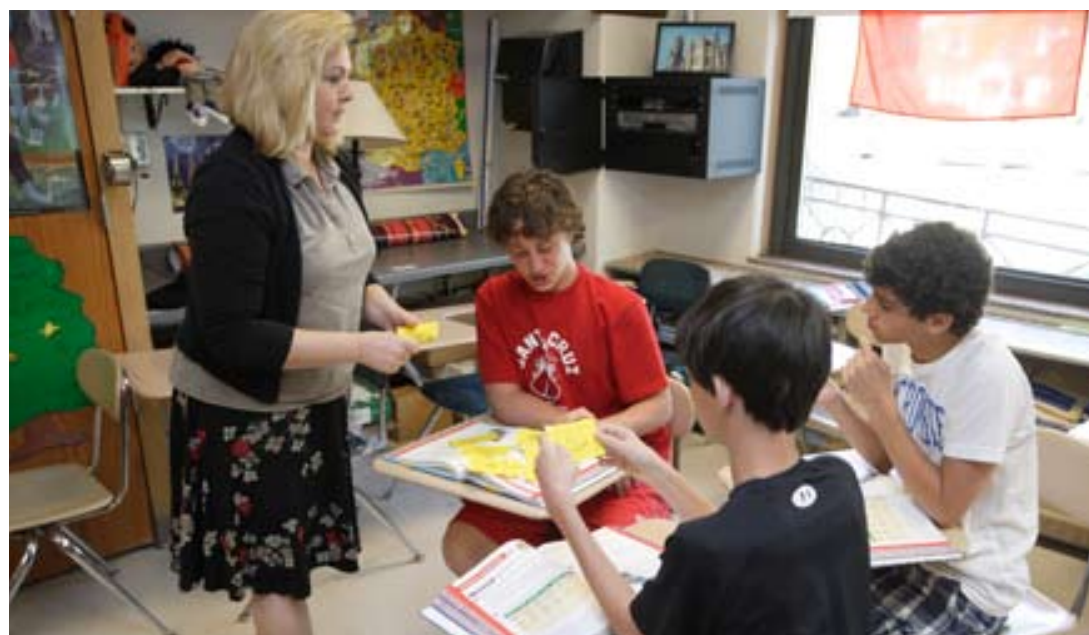

Mathematical concepts embedded in human culture can be studied as they are revealed in literature. photo by Alan Geho

\section{Conclusion}

This article has described a philosophical and pedagogical basis for incorporating mathematical concepts, inquiry, and problem solving into a language arts classroom, as they may naturally fit with the curriculum. Although the form of these discussions varies with each story and especially with grade levelmoving from questions of what and how to why-the underlying tenet remains constant: mathematical concepts should be found and explored as they are encountered in stories and other literature. The goal is to incorporate math in a literature class to build comprehension and enthusiasm and to discover mathematical concepts in literature as they exist so that knowledge built can be relevant, purposeful, and meaningful. As noted earlier, well designed inquiry 
learning produces knowledge that can be widely applied in interdisciplinary contexts. Inquiry requires the learner to turn data and information into useful knowledge, to be actively engaged during the learning process. Problem-based learning provides a context for critical thinking and the development of problem-solving skills. Both reasoning and problem solving are an integral part of all mathematics and language arts learning.

A considerable amount of research has been done recently on the incorporation of literature into mathematics classes, but the reverse incorporation remains relatively unexplored. The ideas discussed and suggested here provide a framework for implementing this kind of curricular integration. Research needs to be done to establish clearly and objectively the extent to which such integration actually improves students' abilities to think critically, as exemplified in their understanding of themes encountered in their reading and their ability to apply the mathematics they know to other contexts in which it naturally arises. However, as some researchers (e.g., Pasko, 2004, and Selfe, Petersen, \& Nahrgang, 1986) have noted in researching matters like this, it is difficult to obtain "quantifiable data that would answer our central questions." First, it is difficult to develop quantifiable measures that really measure the quantities of interest. Second, quantifiable measures may not tell the whole story; qualitative measures such as interviews and observations may be necessary to gain insights about what goes on within the classrooms under study. And, since conclusions are often drawn (e.g., to recommend implementing curricular integration) from statistics, one must consider what kinds of conclusions can be made from non-quantitative measures.

Although considerable thoughtful research is necessary to establish any quantitative edge given by integrating the studies of mathematics and literature when appropriate, there are conclusions that can be drawn at this point. First, there are times when themes that arise in the study of literature have quantitative aspects so that some quantitative literacy and discussion is necessary for a complete appreciation of the work. Second, it is possible to integrate mathematics, literature, and other subjects in a natural way that helps students construct meaning. Of course, as with any sort of teaching innovation or learning practice, students will learn the most through these methods if exposed regularly to them across their years of education. Finally, the way in which the appropriate incorporation of mathematics into the study of literature fits into the current sets of national curricular guidelines justifies a serious exploration of its effectiveness in training students to think critically.

\section{References}

Albanese, M. A., \& Mitchell, S. (1993). Problem-based learning: A review of literature on its outcomes and implementation issues. Academic Medicine, 68(1), 52-81.

Beane, J. A. (1997). Curriculum integration: Designing the core of democratic education. New York: Teachers College Press.

Bintz, W. P., \& Moore, S. D. (2002). Using literature to support mathematical thinking in middle school. Middle School Journal, 34(2), 25-32.

Bransford, J., Brown, A., \& Cocking, R. (Eds.). (1999). How people learn. Washington, DC: National Academy Press.

Burton, D. M. (1997). The history of mathematics, an introduction (3rd ed.). New York: McGraw-Hill.

Engel, J. (1991). Not just a method but a way of learning. In D. Bould \& G. Felletti (Eds.), The challenge of problem-based learning (pp. 21-31). New York: St. Martin's Press.

Hellwig, S., Jacobs, J., \& Monroe, E. (2000). Making informed choices: Selecting children's trade books for mathematics instruction. Teaching Children Mathematics, 7(3), 138-143.

Jacobs, A., \& Rak, S. (1997). Mathematics and literature: A winning combination. Teaching Children Mathematics, 4(3), 156-157.

Jenner, D. M. (2002). Experiencing and understanding mathematics in the midst of a story. Teaching Children Mathematics, 9(3), 167-171.

Kliman, M. (1993). Integrating mathematics and literature in the elementary classroom. Arithmetic Teacher, 40, 318-321.

Melser, N. A., \& Leitze, A. R. (1999). Connecting language arts and mathematical problem solving in the middle grades. Middle School Journal, 31(1), 48-54.

National Council of Teachers of English. (2006). NCTE principles of adolescent literacy reform. Urbana, IL: Author.

National Council of Teachers of Mathematics. (2000). Principles and standards for school mathematics: An overview. Reston, VA: Author.

Pasko, M. (2004). Curriculum connections: Linking literature and math. Networks: An on-line journal for teacher research, 7(2). Retrieved October 18, 2006, from http://education.ucsc.edu/ faculty/gwells/networks/journal/Vol.7(2).2004may/Pasko. html.

Selfe, C. L., Petersen, B. T., \& Nahrgang, C. L. (1986). Journal writing in mathematics. In A. Young \& T. Fulwiler (Eds.), Writing across the disciplines: Research into practice (pp. 192-207). Upper Montclair, NJ: Boynton/Cook.

Spann, M. B. (1992). Multicultural activities: An integrated approach. New York: Scholastic.

Christopher M. Kribs Zaleta is an associate professor of mathematics and curriculum and instruction at the University of Texas, Arlington. E-mail: kribs@uta.edu

Kim K. Ruebel is an assistant professor of curriculum and instruction at the University of Texas, Arlington. E-mail: kruebel@uta.edu 